Received: July 29, 2017

Accepted: August 07, 2017

Published: August 10, 2017

\section{Artificial Brain and "Human-Machine" Integration}

\author{
Chi Tran*
}

\begin{abstract}
The Faculty of Geodesy, Geospatial and Civil Engineering, WM University in Olsztyn, Poland
\end{abstract}

*Corresponding author: Chi Tran, The Faculty of Geodesy, Geospatial and Civil Engineering, WM University in Olsztyn, Poland, E-mail: tran.chi@uwm.edu.pl

\section{Abstract}

We present, in this short paper, a model of artificial brain based on the Software-Hardware integration in the "1 $1=1$ " philosophy framework using machine learning and multiprocessor system on chip, SoC. Its virtual experiences are generated by a deep learning process with random changing of the structure of a net of artificial neural network, NoNN, using Monte Carlo method. It ensures creative property of the human cognitive processing and possibility of the "humanmachine" integration/"Human brain-Artificial Brain" integration, which should be applied in various areas of online control.

\section{Keywords}

Philosophy; Meta-concept; Machine learning; Artificial brain; "Human-Machine" integration; Online control;

\section{Introduction}

$$
" 1+1=1 " \text { ! }
$$

According to Georg Wilhelm Friedrich Hegel (1770-1831), we cannot have the "pure being" unless we also take into consideration "pure nothing" i.e., being and nothing are oneness $(1+$ $1=1$ ) which is neither being nor nothing but a third option presented rather by meta-language, "being-nothing", that transcends both. It we call the "one" philosophy. In fact, the "one" philosophy is find in Indian four-cornered argumentation logic (about 500 BC): affirmation $X$, negation of $X, \neg X$, both $X \wedge \neg X$ and neither $\neg(X \vee \neg X)$, in which, $X \wedge \neg X$ is integration of $X$ and $\neg X$ which represents a third option that transcends both. It describers not knowledge originated from logic (logical knowledge) but rather knowledge originated from a cognitive process in the human brain; in the Chinese "one" philosophy presented by Zhuang Zhou - (369-286 B.C.), in which he claimed that Discrimination, and the opposition of it, Nondiscrimination, are seen as oneness Discrimination-Nondiscrimination. It is not the common sum, Discrimination + Nondiscrimination, but rather an integration described perhaps firstly in the world by meta-language Discrimination-Nondiscrimination referred as a third option that transcends both Discrimination and Nondiscrimination. It represents his feeling comes from cognitive processing; in Levis's modal logic and Lukasiewicz's multivalued logic (1930), in which, the value of truth like the third argumentation of Indian logic, is the integration of true and false - "false-false" integration. It is finding also in the "membership-nonmembership" integration presented in the Lotfi Zadeh's fuzzy set theory (1965). It is clear that various meta-concepts, "being-nothing", "DiscriminationNondiscrimination", "true-false" and "membership-nonmembership" have not meaning associated with common word/words, but another meaning related to the denotative concept described in the "sense and denotation" theory written by Frege FLG (1848-1925), which represents a meaning in the semantic and pragmatic aspects of both conscious and non-conscious thoughts of the human brain [7].

Likewise, according to the "one" philosophy, it is impossible to understand the real world (reality) without the virtual world (virtual reality) contained in mind (in the human brain). That is, when we receive what we consider to be reality, which is a limit to what can be known of the so called objective reality. It is a first perception, which we can describe through common language and Boolean numbers within the syntax constraints and counter-concepts contained in the framework of two-valued logic. We have also relationships to this reality through our brain with chemical, energetic responses and responses of our organsenses. That is to say, we have relationships to other part of reality through high level cognitive responses. It is strictly limited to brain based processing related to an interface for another part of reality. What we get from there, through intuition, experience and process of cognitive information processing, is called second perception. Its description is, sometime, beyond limits of common language and Boolean numbers. This second perception described by meta- language represents the semantic and pragmatic respects of new knowledge in the framework of epistemology including predictive mathematics and modern logic. Thus, concep- 
tion of reality depends really on how to create also a simulation of the cognition and cognitive processing. We are in the state of transitioning to an increasingly mind-dependent viewpoint of reality which represents a necessary integration of the real world and the human brain (the virtual world) - the "reality-virtual reality" integration rather than common connection "reality + virtual reality". It is really the human participation to deal, in the "one" philosophy framework with the "Human-Machine" integration for various fields of online control.

\section{The Real World and Virtual World}

The Internet of Things, IoT, has evolved from the convergence of the Internet, wireless technologies and Micro-ElectroMechanical Systems, MEMS, which contains micro-circuitry on a tiny silicon chip into which some mechanical device such as a sensor has been manufactured. Wireless is a term used to describe telecommunications in which electromagnetic waves/acoustic waves carry the signal over part or the entire communication path called machine to machine, M2M, communication. Generally, the physical signals from sensor are sent to an analog signal processing device in the form of an amplifier or a low-pass filter. On the basis of the information received, the actuators make the necessary decisions about how to respond to the appropriate actions. It creates, however, a new type of data, big data, James [9], Vossen $\mathrm{G}$ [19] - a large number of datasets in the streaming and dynamic forms. Thus, we need a new approach that seeks to discover new information, identify and categorize data, focusing on exploring natural phenomena, acquiring new knowledge, and understanding real-time laws of nature. IoT is an environment where the real world and virtual world are constantly connected via the wireless sensor network, WSN, in which, internet/cloud is referred to as the virtual world. It enables us to observe and control our surroundings anytime, anywhere, which is represented graphically in the following figure 1 .

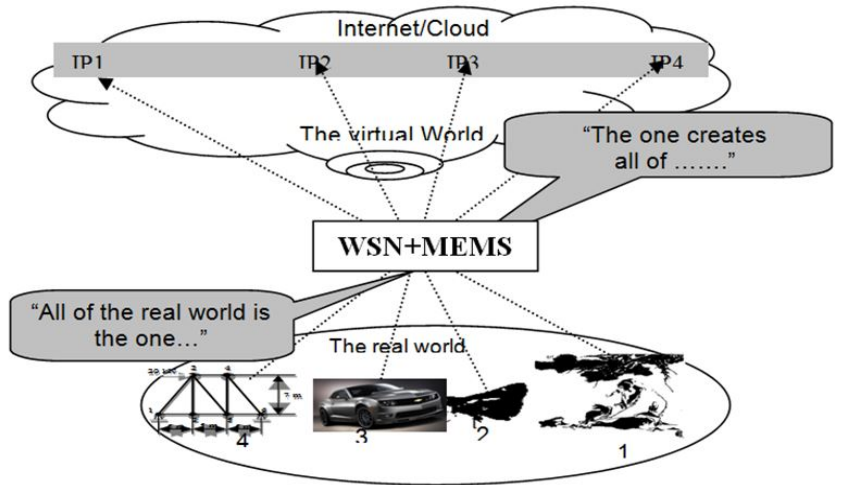

Figure 1: The "real world-virtual world" connection - the Internet of Things, IoT.

On the one hand, the real world is really connected to the human brain (the virtual world) through the organ senses. Thus, the human brain becomes the Brain of Thing, BoT, (the intelligent IoT, iIoT), which is shown in the following figure 2 .

On the other hand, the human brain or brain in short term (B) is still a physical world. It has a virtual world, which should

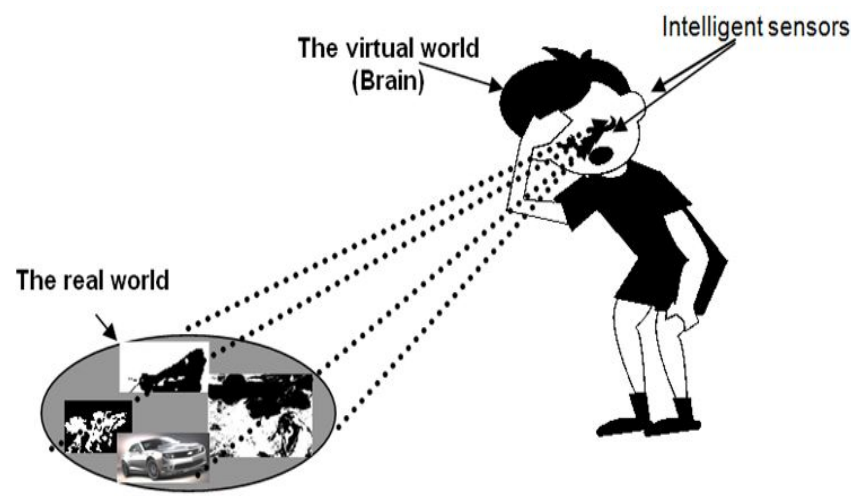

Figure 2: The Brain of Thing, BoT.

be an Artificial Brain/Informatics Brain (AB/IB). Thus, we have like the Internet of Thing the "B (real world) $\mathrm{AB}$ (virtual world)" connection. Collected information about the states of the human brain through brainwaves is transmitted to the artificial brain using brain wearable in order to detect the human thoughts, feelings regardless of the human behavior and to monitor controller's experiences, detect focused thoughts of the human brain [20]. It is not the "B-AB" connection in the common sense but rather the "B-AB" integration in the " $1+1=1$ philosophy" framework, which is so called "Human-Machine" integration. It is neither Human nor Machine but a third option - high level intelligence which is presented in the following figure 3.

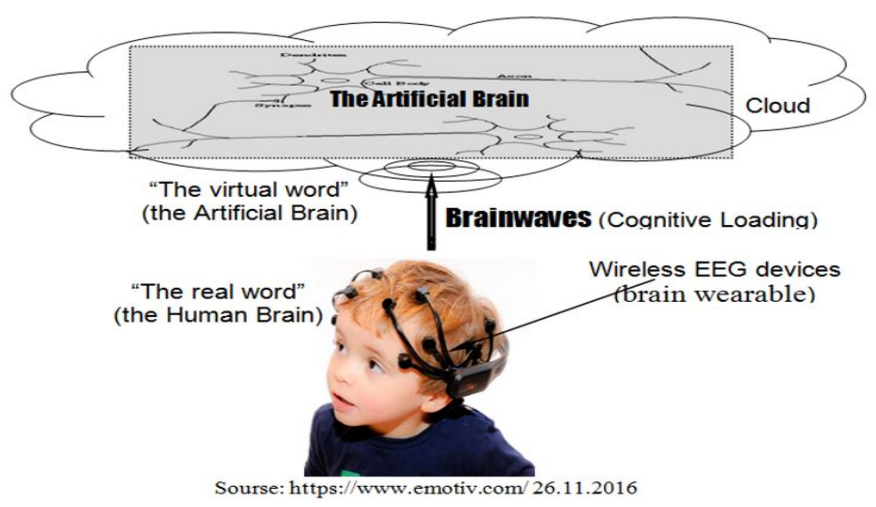

Figure 3: TThe "Brain-Artificial Brain" integration.

It should be used to control physical electronics with mere thoughts and to expand and improve the way our brains is studied and understood.

The Artificial Brain ( $A B$ ), has rather artificial experiences created by learning process using virtual training data and modern informatics in short time. It should be constructed to deal with big data analysis, to support unpredictable queries against cognitive data, to analyze the so-called stream dynamical data in order to discover new insights focused on investigating natural phenomena, acquiring new knowledge, and understanding the laws of nature. To do this, we use Machine Learning (ML) as a software part and MultiProcessorSystem on a chip/system on a chip, SoC, as a hardware component to build an IT model as a software-hardware integration within the "one" philosophy framework, which is presented on the following figure 4. 


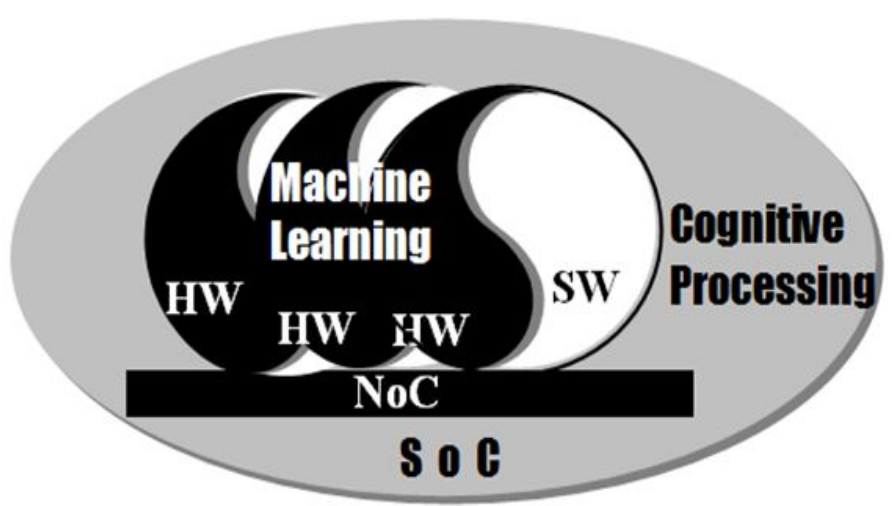

Figure 4: The artificial brain based on the "SW-HW" integration.

\subsection{Hardware}

MultiProcessorSystem on Chip, SoC. Instead of CPU architecture, SoC is a microchip with all necessary electronic circuits and parts for a given system on a single Integrated Circuit, IC - a chip, or a microchip, which includes a Reduced Instruction Set Computer (RISC) [10]. The SoC architecture is specified as a set of processor and hardware subsystems that interact via communication network referred to as Net on Chip, NoC.

The open multi-processing, OpenMP, is a parallel programming model for SoC, a programming interface that supports multiplatform shared memory multiprocessing programming. It assumes a shared memory model with all the threads having access to the same globally shared memory. The OpenMP consists of a set of compiler directives to define a parallel region. We can write an OpenMP program by inserting OpenMP directives (parallel loop, work is distributed) into a serial program, for example,

\#programa omp parallel for

for $(i=0 ; i<100 ; i++)$

\{

$\operatorname{Data}[i]=0$;

\}

Cognitive Chip, CC. The SoC technology is developed using neurosynaptic chip/Cognitive Chip (CC) or a microprocessor that functions like more the human brain than a common CPU does. It is made to function like the human brain on the hardware rather than software level used to imitate thoughts and learn of the human brain. IBM's brain-inspired architecture consists of a network of neurosynaptic cores, which are distributed and operate in parallel. According to IBM's study, a chip of one million neuron brain-inspired processor is capable of 46 billion synaptic operations per second.

IBM's Supercomputer. Researches from IBM create computing system - new supercomputer used to simulate and emulate the brain's abilities for sensation, perception, action, interaction and cognition with compact size. The new supercomputer, according to IBM research lab, will consist of 64 million neurons and 16 billion synapses. A single processor in the system consists of 5.4 billion transistors organized into 4096 neural cores, creating an array of 1 million digital neurons that communicate with one another via 256 million electrical synapses. The architecture is specified as a set of cores (processors) and hardware subsystems that interact via communication network including local instruction and data bus.

\subsection{Software}

The parallelization mechanism is called partitioning, which will be mapped on the target architecture. The parallelization of the application consists in dividing the computation in several pieces that can be executed in parallel. These different pieces group several functions of the application are named tasks or processes. The mapping represents the association between the tasks and the processing elements on which they are executing, and the association between the buffers used for the communication between the tasks and the hardware. For example, we can use the analysis of big data through Apache's Hadoop using Google's MapReduce. It, in particular, the Hadoop Distributed File System (HDFS) is data storage component of the open source Apache's Hadoop project which had evolved for excellent offline data processing platform [18,21].

Software based MapReduce is a programming model and the Google's software framework for large-scale distributed computing on large amounts of data in order to collect and to mine, e.g., satellite and thermal imagery, and other readily available information. Given a set of data, a series of operations (kernel functions) is applied to each element in the dataset and local onchip memory is reused to minimize external memory bandwidth and to automate and optimize on-chip management "map-reduce" process.

Machine Learning provides computers with an ability to learn without programming. Various approaches are being taken to formulate a new field of so-called machine learning. According to Corinna Cortes, et al. [4], a new type of machine learning (support-vector network) is constructed. It implements the following idea: mapping the input vectors into some high dimensional feature space $\mathrm{Z}$ through some non-linear mapping chosen a priori, constructing a linear decision surface with special properties that ensure high generalization ability of the network. Neural networks are a common type of another Machine Learning, ML, that tries to mimic, how neurons interrelate in the human brain to pass information around. An attempt to emphasize the neurobiological aspects of artificial neural computation is presented by different authors, [2,3,5]. It focuses on the development of an artificial brain that can learn to develop own experience and change under the influence of new data. It can be used to detect patterns in data and adjusts program actions accordingly. ML not only enables us to understand the notion of the real world, but enables us to learn and change its own future behavior also. Also, how we can overcome unwanted situations while building NN models is presented by Tavish Srivastava [17].

We present in this paper a combination, according to "SW-HW" integration, of a net of multilayer feedforward neural networks FFNN, with sigmoidal neurons, and SoC used for simulation of an inductive learning process[14]. In which, we use the Resilient Back Propagation, RBP, training algorithm in order to eliminate 
the harmful effects of the magnitudes of the partial derivatives.

Training data is the one problem of machine learning, in which we have to generate sufficient enough of a number of training data with suitable distribution within the problem domain. Before we start learning, we need a lot of training data, but we usually do not have all the data available to train the computer for learning. Training data has a significant effect on the learning and generalization performance of a network. Depending on studied problem, for the "human-machine" integration for example, training data set is created by an available benchmark database of EEG data labeled with emotions. While in the structural mechanics, training data/virtual data is created by integration of Monte Carlo Simulation and Finite Element Method (MCSFEM). We perform, by this way, a combination of highly creative mathematical possibilities of Monte Carlo simulation and high accuracy in the mechanical aspects of FEM.

Least Squares Error, LSE, is an example of supervised training, in which the learning rule is provided with a set of examples, $\mathrm{n}$ inputs, $\mathrm{p}_{n}$, and $\mathrm{n}$ target outputs, $t_{n}$, to the network of desired behavior:

$$
\left\{\mathrm{p}_{1}, \mathrm{t}_{1}\right\},\left\{\mathrm{p}_{2}, \mathrm{t}_{2}\right\}, \ldots,\left\{\mathrm{p}_{\mathrm{n}}, \mathrm{t}_{\mathrm{n}}\right\}
$$

The error is calculated as the difference between the target output, $\mathrm{t}$, and the network output, a, in order to minimize the average of the sum of these errors:

$$
M S E=\frac{1}{N} \sum_{n=1}^{N} e(n)^{2}=\frac{1}{N} \sum_{n=1}^{N}(t(n)-a(n))^{2}
$$

The LSE algorithm adjusts the weights and biases of the linear network so as to minimize this mean square error, MSE.

Support Vector Machine, SVM, have been introduced for solving pattern recognition problems. JAK Suykens, J Vandewalle [16] formulate a least squares version of support vector machine, SVM, for classification problems with two classes. It is based on Vapnik-Chervonenkis theory and structural risk minimization principle. Authors claimed that a least squares SVM with Gaussian radial basis function, $\mathrm{RBF}$, kernel is readily found with excellent generalization performance and low computational cost. Many algorithms are introduced, which are employed to classify the data into emotions, see Lin et al. [11] or Murugappan et al. [13]. It indicates that we can create an ability to recognize the human emotions from elektroencefalografia, (EEG) which is a non-invasive diagnostic method used to study bioelectrical brain activity from the scalp in order to approximate a level of the human brain intelligence.

Support vector machines for classification, and least squares support vector machines, SVM, is a powerful methodology for solving problems in nonlinear classification, in which, function and density estimation has led to other recent developments in kernel based methods. It has been introduced within the context of statistical learning theory. In these methods one solves convex optimization problems, typically by quadratic programming. Least squares support vector machines, LS-SVM, is reformulations to the standard SVM. In which, the cost function is a regularized least squares function with equality constraints, leading to linear Karush-Kuhn-Tucker systems. That is, given a training set of $\mathrm{n}$ data points:

$$
\left\{\mathrm{p}_{\mathrm{k}}, \mathrm{t}_{\mathrm{k}}\right\}, \mathrm{k}=1, \ldots, \mathrm{N}, \mathrm{p}_{\mathrm{k}}, \mathrm{t}_{\mathrm{k}} \in R^{n}
$$

Where, $\mathrm{p}_{\mathrm{k}}$ and $\mathrm{t}_{\mathrm{k}}$ are the $\mathrm{k}^{\text {th }}$ input pattern and the $\mathrm{k}^{\text {th }}$ output pattern respectively. The support vector method approach aims to construct a classifier in the form:

$$
t(p)=\operatorname{sign}\left(\sum_{k=1}^{N} \alpha_{k} t_{k} \psi\left(p, p_{k}\right)+b\right)
$$

Where $\alpha_{k}$ are positive real constants and b is a real constant. $\psi$ is selected function by user, e.g., linear SVM, RBF SVM or two layer neural SVM etc.. The classifier is constructed as:

$$
t_{k}\left[w^{T} \varphi\left(p_{k}\right)+b\right] \geq 1, k=1, \ldots, N
$$

Where, $\varphi$ is a nonlinear function which maps the input space into a higher dimensional space; $w$ is the weight vector in primal weight space. The risk bound is minimized by formulating the optimization problem, which represents the structural risk minimization principle:

$$
\underset{w, \xi_{k}}{\operatorname{Min} J}\left(w, \xi_{k}\right)=\frac{1}{2} w^{T} w+c \sum_{k=1}^{N} \xi_{k}
$$

Where, $\xi$, e are error variables, c is a constant. Many authors introduce a least squares version to the SVM classifier by formulating the classification problem as:

$$
\underset{w, b, e}{\operatorname{Min} J}(w, b, e)=\frac{1}{2} w^{T} w+\frac{1}{2} \gamma \sum_{k=1}^{N} e_{k}^{2}
$$

Subject to the equality constraints

$$
t_{k}\left[w^{T} \varphi\left(p_{k}\right)+b\right]=1-e_{k}, k=1, \ldots, N
$$

In this method one maps the data into a higher dimensional input space and constructs an optimal separating hyperplane in this space. In which, kernel functions are chosen such that a bound on the 'Vapnik-Chervonenkis'dimension is minimized. In the research presented by Francesc Pozo et al. [6] and Ramin Ghiasi, et al. [15], authors construct a new combinational wavelet kernel using the multidimensional orthogonal modified LittlewoodPaley wavelet and thin plate spline radial basis function kernel. A novel combinational kernel function, namely the TPSLPW is constructed by combining two kernel functions as follows

$$
\begin{aligned}
& K_{\text {TPSLPW }}\left(p_{k}, p_{l}\right)=\rho K_{\text {ThinPlateSpline }}\left(p_{k}, p_{l}\right)+(1-\rho) K_{L-P_{\text {wavelet }}}\left(p_{k}, p_{l}\right) \\
& K_{\text {TPSLPW }}\left(p_{k}, p_{l}\right)=\rho\left\|p_{k}-p_{l}\right\|^{2} \ln \left\|p_{k}-p_{l}\right\|+ \\
& \quad+(1-\rho) \prod_{i=1}^{N} \frac{1}{\pi \sqrt{q-1}} \frac{\sin (q \pi)\left(\frac{\left(p_{k}\right)_{i}-\left(p_{l}\right)_{i}}{a_{i}}\right)-\sin (\pi)\left(\frac{\left(p_{k}\right)_{i}-\left(p_{l}\right)_{i}}{a_{i}}\right)}{\left(\frac{\left(p_{k}\right)_{i}-\left(p_{l}\right)_{i}}{a_{i}}\right)}
\end{aligned}
$$

Where, $\mathrm{q}>1$ and $0 \leq \rho \leq 1$ are a band-scalars and $a_{i}$ is the flexible coefficient of wavelet, $a_{i}>0$; K(.) denotes a kernel.

Resilient back propagation (RBP) training presented by Mar- 
tin Riedmiller et al. [12], can be used in order to eliminate harmful effects of the magnitudes of the partial derivatives in order to overcome the inherent disadvantages of pure gradient-descent. Only the sign of the derivative is used to determine the direction of the weight update. Size of the weight change is determined by a separate update value. Authors introduce for each weight its individual update-value, $\triangle_{i j}$, which solely determines the size of the weight-update. This adaptive update-value evolves during the learning process based on its local sight on the error function $\mathrm{E}$, according to the following learning rule:

$$
\Delta_{i j}^{(t)}=\left\{\begin{array}{c}
\eta^{+} . \Delta_{i j}^{(t-1)}, \text { if } \frac{\partial E^{(t-1)}}{\partial w_{i j}} \cdot \frac{\partial E^{(t)}}{\partial w_{i j}}>0 \\
\eta^{-} \cdot \Delta_{i j}^{(t-1)}, \text { if } \frac{\partial E^{(t-1)}}{\partial w_{i j}} \cdot \frac{\partial E^{(t)}}{\partial w_{i j}}<0 \\
\Delta_{i j}^{(t-1)}, \text { else }
\end{array}\right.
$$

Where $0<\eta^{-}<1<\eta^{+}$. Once the update-value for each weight is adapted, the weight-update it follows a simple rule:

$$
\Delta w_{i j}^{t}=\left\{\begin{array}{l}
-\Delta_{i j}^{(t)}, \text { if } \frac{\partial E^{(t)}}{\partial w_{i j}}>0 \\
+\Delta_{i j}^{(t)}, \text { if } \frac{\partial E^{(t)}}{\partial w_{i j}}<0 \quad ; w_{i j}^{(t+1)}=w_{i j}^{(t)}+\Delta w_{i j}^{(t)} \\
0, \text { else }
\end{array}\right.
$$

With one exception:

$$
\Delta w_{i j}^{(t)}=-\Delta w_{i j}^{(t-1)}, \text { if } \frac{\partial E^{(t-1)}}{\partial w_{i j}} \cdot \frac{\partial E^{(t)}}{\partial w_{i j}}<0
$$

Using, in this paper, two keener functions: Hyperbolic tangent sigmoid, respectively:

$$
\operatorname{tansig}(\mathrm{n})=2 /(1+\exp (-2 * \mathrm{n}))-1 ; \log \operatorname{sig}(\mathrm{n})=
$$

$1 /(1+\exp (-n))$,

This allows for all weights of the neural network to adapt in order to minimize the error on a set of vectors belonging to pattern recognition problems.

\subsection{Software-Hardware Integration}

In fact, SW and HW are undoubtedly more sophisticated than some years ago. The SW-HW integration, in deep idea of the "1 + $1=1$ "philosophy, is what far more than the sum, SW + HW, separately. It creates (1) the so called MapReduce based on "SW-HW" integration rather than the SW based MapReduce; (2) Learning based on "SW-HW"integration which are presented bellows.

MapReduce based on "SW-HW"integration is new form using SoC rather than SW based MapReduce. It will parse the input, splits to each slave (core of SoC) and produces records. Intermediate results generated in the map phase are sorted by MapReduce, entered into slaves and next into the reduction phase. This MapReduce connected with program openMP is shown in the fol- lowing figure 5 .

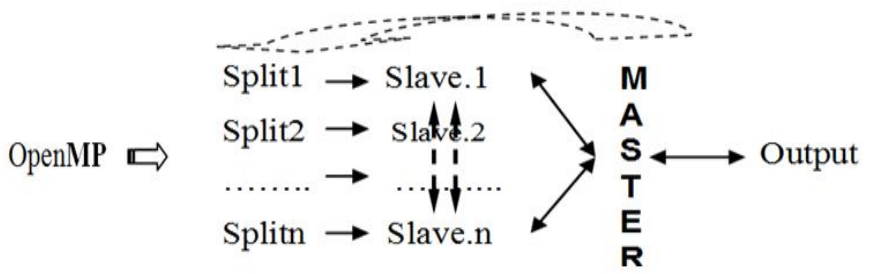

Figure 5: Map Reduce based on "SW-HW" integration using SoC.

Learning based on "SW-HW"integration. Multilayer neural networks can have several layers in all cases, but it is not obvious how many neural layers and neuron numbers of each to include in the network. Generally, NN consists of interconnected processing elements called nodes or neurons that work together to produce an output function. We have NN architecture presented by: $\mathrm{p}_{i}$ $m_{j}-\mathrm{o}_{k}-\mathrm{W}_{j} ; \mathrm{i} \in \mathrm{I}$ (input number), $\mathrm{j} \in \mathrm{J}$ (layer number); $\mathrm{k} \in \mathrm{K}$ (output number); where, $\mathrm{p}_{i}$, denotes input data; $m_{j} \in \mathrm{M}$ (neuron number in each layer); $\mathrm{o}_{k}$ - output number; $W_{j}$ denotes weigh. The teaching of the human brain is represented by continuous changes in the parameters $\mathrm{p}, \mathrm{m}, \mathrm{o}, \mathrm{w}$, in the permissible spaces I, $\mathrm{J}, \mathrm{K}, \mathrm{W}$ respectively of the thought function to achieve any goals:

$$
\text { Thought }=\forall_{i \in I, j \in J, k \in K, w \in W} f\left(p_{i}, m_{j}, o_{k}, W_{j}\right)
$$

Changes of these arguments in the random way ensure the creativity of the human unconscious thought that provides not only experience but also special capability of the human brain such as creation and intuition. For example, the artificial experience is generated by a learning process in short time using virtual training data. It is originated from a number of $\mathrm{NN}$, connected by net on chip, NoC, which we call a net of NN, NoNN. Two neural layers for feedforward network are accepted and large number of bigdata processing are used to search an optimal NN structure, which is formulated such that during training using SIMD technology, neural network structure is changing continuously and the weights of the network are iteratively adjusted in order to minimize the network performance function, MSE $<5 * 10-6$ [8]. For layered feedforward network with equality: input number $=$ output number $=$ number of neurons of second layer $=\mathrm{q} ; \mathrm{m}$ denotes neuron number of first layer; $M$ denotes the acceptable neurons number (a fixed number of first layer, $M=50$ for example); $\mathrm{j}$ denotes layer number, $\mathrm{j} \in \mathrm{L}$; $\mathrm{n}$ - a number of slaves, $\mathrm{n} \in \mathrm{N}$ (NN number). Then, q-m-q-q is referred as NN architecture. An optimal solution of the NoNN is presented by:

$\operatorname{Min}(M S E)=\exists_{q-m-q-q} \operatorname{Min}_{n \in N}\left\{\begin{array}{l}\min _{m \in M, j \in L}\left(M S E_{j}\right)_{1}, \min _{m \in M, j \in L}\left(M S E_{j}\right)_{2} \\ , \ldots, \min _{m \in M, j \in L}\left(M S E_{j}\right)_{n}\end{array}\right\}$

Search of optimal architecture (good enough) is shown in the figures $6 a, b$.

Machine Learning is essentially a system of machines outfitted with data-collecting technologies so that those machines can com- 


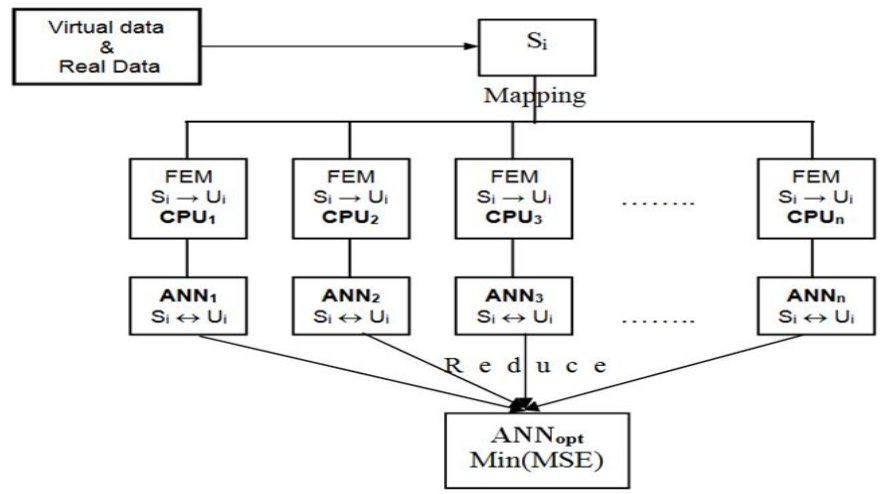

Figure 6a: Deep learning of NoNN based on the "HW-SW" integration.

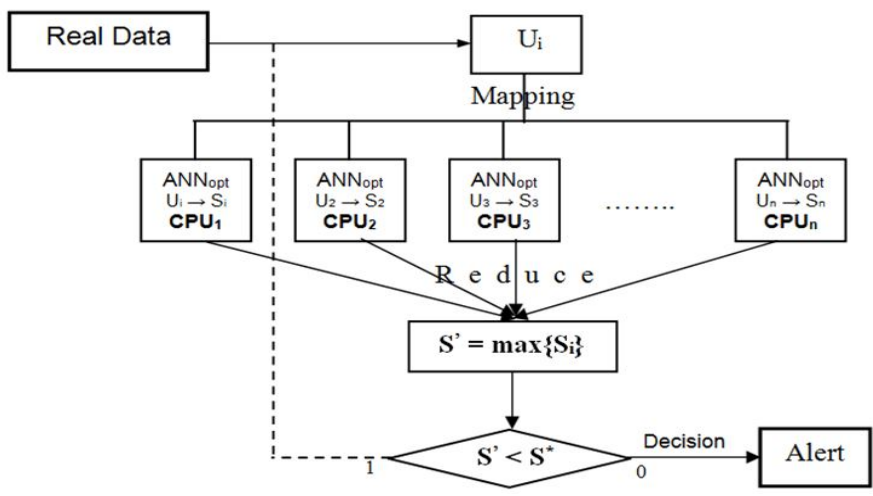

Figure 6b: To make an optimal decision in the framework of the "HW-SW" integration.

municate with one another. ML functions in the SoC, in which, different NNs are distributed in different cores connected by net on chip, NoC, referred to as interface. In this paper, we want to make a learning program as flexible as possible with respect to the configuration of the SoC architecture, including $n=8$ processors referred as 8 slaves. Determining the optimal architecture of NN through deep learning using NoNN and the integration of Monte Carlo and Finite Element Method, MCSFEM, based on the "SW-HW" integration is shown in the following figure 7.

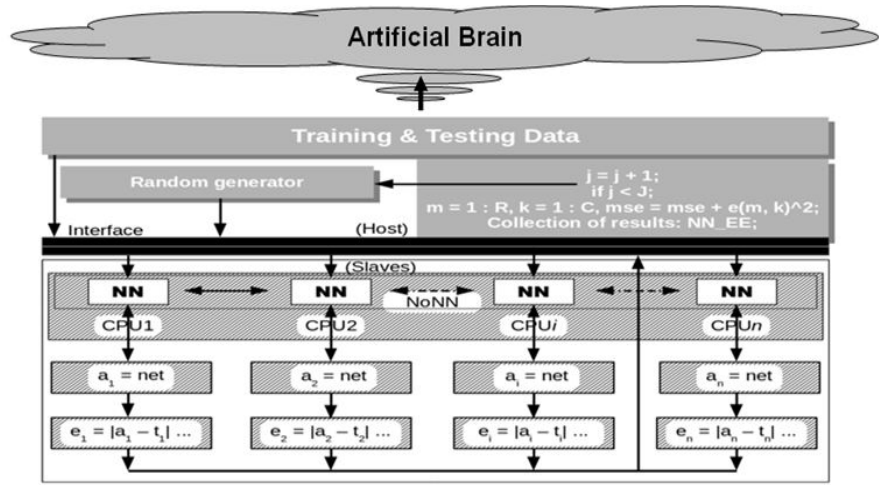

Figure 7: Machine Learning based on the platform of the System on Chip, SoC.

It should be noted that the training of CC and IBM supercomputers will increase processing speed and significantly increase the changing possibility of the number of NN layers as well as the number of neurons in each layer and values of weights, resulting in more accurate and more creative effects.

The "Bridge-Car" Talking Application. For example, the structures equipped with sensors to its monitor in order to communicate the information via the wireless internet to cars. This connection from sensors connected directly to internet/cloud, and then to actuator of the car through the wireless internet, turns critical information to make decision.

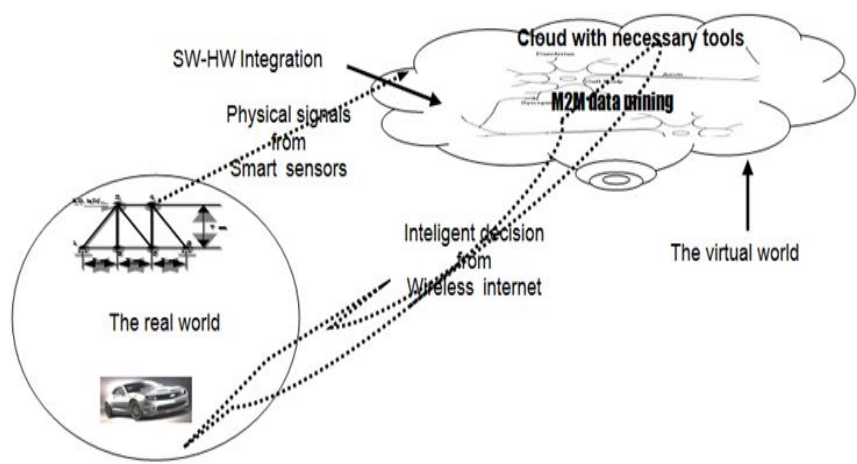

Figure 8: The conversation between the bridge and car - Online control.

\section{4 "Human Brain-Artificial Brain" Integration}

As you see, the human brain, B, has not computational capability for big data as the artificial brain, while the artificial brain has no capability to deal with uncertainties as the human brain can do. We need the human participation for cognitive information processing, which creates a new field of the so called humanmachine integration. It is interaction-based systems - an open challenge of online control.

Human-Machine Integration Application. A new idea of PUMA 560 robot control, through concentration of thoughts, using the combination of wireless EEG devices and the artificial brain containing the virtual experiences after training, is shown in the figure 9.

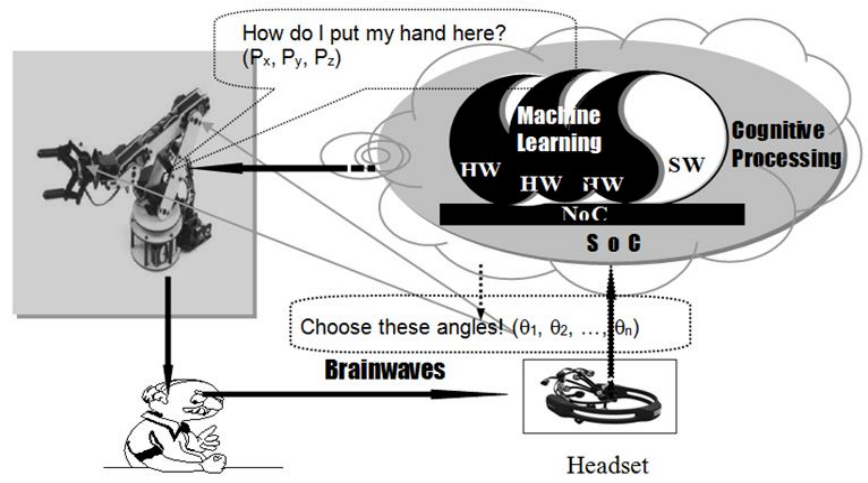

Figure 9: Human-Machine connectivity in the online control.

It represents the Human-Machine integration (the "B-AB" integration) using ML based on the platform of the SoCC (System on Cognitive Chip). 


\section{Conclusions and Discussion}

It indicates that human intelligence is based on not only human experiences derived from many years of live but also analysis in real time of new event, based on random changes of the NN architecture itself without our consciousness - It works on itself. It is formulated using a deep learning process, in which, an intelligent decision will be reached by machine learning and random changes continuously of NN architecture of a net of neural network NoNN.

Finely, the "B-AB" integration helps us to get the so called high level intelligence, which should be applied in new areas of online control and others.

Work in the future. Because the human brain not only collects and analyzes information from the senses passively, but also actively introduces without conscious reasoning. the hypothesis used to control the sensory activity reversely. It is special capability of the human brain which we call the human intuition, creativity. By the way presented above utilizing intuitive logic and IBM's supercomputer, we would like to develop a computational model used to find full solutions of the thought function in order to mimic those brain abilities.

\section{References}

1. Krizhevsky A, Sutskever I, Hinton GEl. Imagenet classification with deep convolutional neural networks. In NIPS. 2012;1(2):4.

2. Gruning A, Bohte SM. Spiking Neural Networks: Principles and Challenges. European Symposium on Artificial Neural Networks, Computational Intelligence and Machine Learning. 2014.

3. Gardner B, Gruning A. Learning temporally precise spiking patterns through reward modulated spike-timing-dependent plasticity. International Conference on Artificial Neural Networks (ICANN). 2013:256-263.

4. Corinna C, Vapnik V. Support-Vector Networks. Machine Leaming. 1995;20(3):273-297.

5. Dauce E. Toward STDP-based population action in large networks of spiking neurons. ESANN 2014 proceedings, European Symposium on Artificial Neural Networks, Computational Intelligence and Machine Learning. 2014.

6. Francesc P, Ignacio A, Luis EM, Magda R, Elena P. Detection of structural changes through principal component analysis and multivariate statistical inference. Structural Health Monitoring. 2016;15(2):127-142.

7. Frege FLG. 'Uber Sinn und Bedeutung', in Zeitschrift fÃijr Philosophie und philosophische Kritik. 1980;100:25-50. Translated as 'On Sense and Reference' by M. Black in Translations from the Philosophical Writings of Gottlob Frege, P. Geach and

M. Black (eds. and trans.), Oxford: Blackwell, third edition

8. Jih-Ching C, Yu-Liang C. A multi-streaming SIMD multimedia computing engine. Microprocessors and Microsystems. 2010;34(7-8): 247-258.

9. James MT. Big Data: Unleashing information. Journal Syst Sci and Syst. 2013;22(2):127-151.

10. Katalin P, Frederic R, Ahmed AJ, Marilyn W. Embedded Software Design and Programming of Multiprocessor System-onChip. Embedded Systems. 2010.

11. Lin YP, Wang $\mathrm{CH}$, Wu TL, Jeng SK, Chen JH. EEG-based emotion recognition in music listening: A comparison of schemes for multiclass support vector machine. In: ICASSP, IEEE International Conference on Acoustics, Speech and Signal Processing. 2009:489-492. DOI: 10.1109/ICASSP.2009.4959627

12. Martin R, Ileinrich B. A direct adaptive method for faster backpropagation learning. IEEE International Conference on Neural Network. 1993.

13. Murugappan M, Rizon M, Nagarajan R, Yaacob S, Zunaidi I, Hazry D. Lifting scheme for human emotion recognition using EEG. International Symposium on Information Technology. 2008. DOI: 10.1109/ITSIM.2008.4631646

14. Neural Network Toolbox User's Guide. MATLAB v. 2010.

15. Ramin G, Peyman T, Mohammad N. A machine-learning approach for structural damage detection using least square support vector machine based on a new combinational kernel function. Structural Health Monitoring. 2016:1-15.

16. Suykens JAK, Vandewalle J. Least Squares Support Vector Machine Classifiers. Neural Processing Letters 9. 1999:293-300.

17. Srivastava T. 8 Reasons Why Analytics / Machine Learning Models Fail To Get Deployed. 2016.

18. Tom W. Hadoop: The Definitive Guide. OâĂŹReilly Media; 2009.

19. Vossen G. Big data as the new enabler in business and other intelligence. Vietnam J Comput Sci. 2014;1(1):3-14. doi:10.1007/s40595-013-0001-6

20. A headset that reads your brainwaves. Available from: http://www. ted.com/talks/tan le

21. http://hadoop.apache.org/docs/r1.2.1/index.html. 2013. 